\title{
Hepatotoxicidad: patrón colestásico inducido por fármacos
}

\section{Hepatotoxicity: A Drug-Induced Cholestatic Pattern}

\author{
Laura Morales M.,' ${ }^{1}$ Natalia Vélez L., ${ }^{1}$ Octavio Germán Muñoz M., MD. ${ }^{2}$
}

\footnotetext{
Estudiante de medicina, facultad de medicina, grupo de gastrohepatología, Universidad de Antioquia. Medellín, Colombia.

2 Médico internista hepatólogo, Hospital Pablo Tobón Uribe. Grupo de gastrohepatología, Universidad de Antioquia. Medellín, Colombia.

\begin{abstract}
Resumen
La enfermedad hepática inducida por fármacos (DILI) es una condición poco frecuente; sin embargo, explica el $40 \%-50 \%$ de las insuficiencias hepáticas agudas. Su patrón es colestásico en un $20 \%-40 \%$, causado por la inhibición de los transportadores que regulan la síntesis biliar; esta reducción en la actividad es mediada directa o indirectamente por los medicamentos y sus metabolitos, por polimorfismos genéticos y otros factores de riesgo del paciente. Sus manifestaciones van desde las alteraciones bioquímicas en ausencia de síntomas, hasta la insuficiencia hepática aguda y el daño hepático crónico. Aunque no existe un examen o marcador que indique el diagnóstico absoluto de la enfermedad, se han desarrollado escalas y algoritmos que permiten valorar la probabilidad de DILI colestásica, y otras pruebas que por su complejidad y costo no son de uso rutinario. Por lo anterior, es principalmente un diagnóstico de exclusión basado en evidencia circunstancial.

El patrón colestásico de DILI presenta una mejor tasa de supervivencia general pero un mayor riesgo de desarrollo de enfermedad hepática crónica. En la mayoría de los casos, el cuadro del paciente mejora con el retiro del medicamento responsable del daño; la hemodiálisis y el trasplante deben considerarse solo para casos selectos. No se ha probado la eficacia de otras terapias.

En este artículo se profundizará en la fisiopatología, la presentación clínica, bioquímica e histopatológica, además del diagnóstico, manejo y pronóstico de este tipo de colestasis.
\end{abstract}

\section{Palabras clave}

Colestasis, enfermedad hepática inducida por medicamentos, hepatopatía, medicamentos.

\footnotetext{
Abstract circumstantial evidence. selected cases. The effectiveness of other therapies is unproven.

\author{
Keywords \\ Cholestasis, drug, liver disease, drug-induced liver disease.
}

Although drug induced liver disease is a rare condition, it explains $40 \%$ to $50 \%$ of all cases of acute liver failure. In $20 \%$ to $40 \%$ of the cases, the pattern is cholestatic and is caused by inhibition of the transporters that regulate bile synthesis. This reduction in activity is directly or indirectly mediated by drugs and their metabolites and/or by genetic polymorphisms and other risk factors of the patient. Its manifestations range from biochemical alterations in the absence of symptoms to acute liver failure and chronic liver damage.

Although there is no absolute test or marker for diagnosis of this disease, scales and algorithms have been developed to assess the likelihood of cholestatic drug induced liver disease. Other types of evidence are not routinely used because of their complexity and cost. Diagnosis is primarily based on exclusion using

Cholestatic drug induced liver disease has better overall survival rates than other patters, but there are higher risks of developing chronic liver disease. In most cases, the patient's condition improves when the drug responsible for the damage is removed. Hemodialysis and transplantation should be considered only for

This article will delve into the pathophysiology, biochemistry, and histopathology and the clinical presentation of the disease and will discuss diagnosis, management and prognosis of this type of cholestasis. 


\section{INTRODUCCIÓN}

La enfermedad hepática inducida por medicamentos (DILI, por sus siglas en inglés [Drug Induced Liver Injury]) es una causa poco frecuente de este tipo de enfermedad en la población general, dando cuenta de menos del $1 \%$ de los pacientes hospitalizados con ictericia $(1,2)$, y de hasta el $1 \%$ de los pacientes en manejo por medicina interna (principalmente por tuberculostáticos y antineoplásicos) (3); sin embargo, esta entidad explica entre el 40\%-50\% de las causas de insuficiencia hepática aguda (4), y se estima que a los 6 meses del inicio del cuadro clínico, 1 de cada 10 pacientes muere o requiere trasplante hepático, y 1 de cada 5 desarrollan enfermedad hepática crónica (5). Por lo anterior, esta entidad es un importante campo de investigación y vigilancia farmacológica $(4,6)$.

Se han definido 3 patrones de lesión de DILI: colestásico, hepatocelular y mixto. El primero se caracteriza por una elevación de la fosfatasa alcalina (ALP) 2 veces mayor del límite superior normal y/o un $\mathrm{R}$ menor o igual de 2 (siendo R la relación entre ALT y ALP, como se evidencia en la figura 1). El patrón hepatocelular se define como ALT 2 veces mayor del límite superior normal y/o R mayor de 5; y el patrón mixto es ALT 2 veces mayor del límite superior normal, con R entre 2 y 5 (7-10).

$$
R=\frac{\text { ALT del paciente/Límite superior normal de ALT }}{\text { ALP del paciente/Límite superior normal de ALP }}
$$

Figura 1. Definición de $R$ para patrones de DILI. ALP: fosfatasa alcalina; ALT: alanino aminotransferasa.

El 20\%-40\% de los casos de DILI se presentan con patrón colestásico, en comparación con un $40 \%-78 \%$ para el hepatocelular y un $12 \%-20 \%$ para el mixto $(7,10)$; la presentación colestásica, a pesar tener una mejor tasa de supervivencia en general, tiene también menos tasa de progresión favorable del perfil bioquímico hepático y mayor riesgo de desarrollo de enfermedad hepática crónica (11).

En las últimas décadas se han realizado múltiples estudios para identificar los principales factores de riesgo en el desarrollo de toxicidad hepática, al igual que para su diagnóstico oportuno y manejo adecuado; esta revisión resume los aspectos fisiopatológicos, clínicos, diagnósticos y de tratamiento más relevantes de la enfermedad colestásica inducida por medicamentos.

\section{FISIOLOGÍA}

La secreción biliar es un proceso osmótico que se lleva a cabo por la concentración de sales y otros componentes en el canalículo biliar. El transporte de solutos de la sangre a la bilis se hace por medio de los sistemas de transporte en la superficie de las membranas plasmáticas basolateral (sinusoidal) y apical (canalicular) de los hepatocitos (12).

La membrana basolateral contiene las bombas $\mathrm{Na}^{+} /$ $\mathrm{K}^{+}$-ATPasa y los canales de $\mathrm{K}^{+}$que generan un potencial eléctrico transmembrana de aproximadamente $-35 \mathrm{mV}$, el cual mantiene los gradientes iónicos intra y extracelulares y la homeostasis del $\mathrm{pH}$. Este potencial es el que permite la captación de sales biliares conjugadas (ácidos biliares) desde la sangre por medio del cotransportador $\mathrm{Na}^{+} /$taurocolato (NTCP), principalmente (13). Por el contrario, las sales biliares no conjugadas, los aniones orgánicos y muchos otros componentes que se unen a la albúmina son transportados desde el plasma hasta los hepatocitos por sistemas de transporte independientes de $\mathrm{Na}^{+}$, como el polipéptido transportador de aniones orgánicos (OATP) (12).

La excreción canalicular de ácidos biliares (la mayor fracción de solutos orgánicos en la bilis) es mediada por la familia de transportadores dependientes de ATP para ácidos biliares y aniones orgánicos, los cuales representan el paso determinante de la velocidad de formación de la bilis (14). La excreción osmótica activa de ácidos biliares se sigue por el movimiento de agua a través de acuaporinas $y$ uniones estrechas, representando un flujo biliar dependiente de los ácidos biliares; adicionalmente a su actividad osmótica, estos promueven la secreción canalicular de fosfolípidos y colesterol para la formación de micelas biliares mixtas $(13,18)$. También hay compuestos independientes del flujo de ácidos biliares, como el glutatión reducido y el bicarbonato. Tanto los hepatocitos como los colangiocitos secretan y absorben diferentes componentes que modifican las características de la bilis a lo largo de su paso por la vía biliar (figura 2) (15).

El sistema de transportadores hepatobiliares se regula en niveles transcripcionales y postranscripcionales, por ejemplo a través de la activación de ligandos de receptores nucleares; estos hacen la regulación positiva y negativa de las vías de formación biliar, como la de las enzimas detoxificadoras y las bombas de exportación de compuestos biliares, tanto en estado fisiológico como patológico (16) ABCB11. Los componentes biliares, los productos lipídicos, las hormonas y los xenobióticos activan los receptores nucleares como ligandos endógenos o exógenos y modifican de forma coordinada la expresión de genes que codifican los transportadores hepatobiliares y las enzimas de metabolismo de fase I y II (16)ABCB11.

Hay evidencia creciente de que la actividad de los receptores nucleares, como el FXR (receptor X de fernesoide), se afecta mediante la remodelación de la cromatina por medio de la acetilación de las histonas (16)ABCB11. Esto es importante, ya que este es el receptor nuclear mejor defi- 


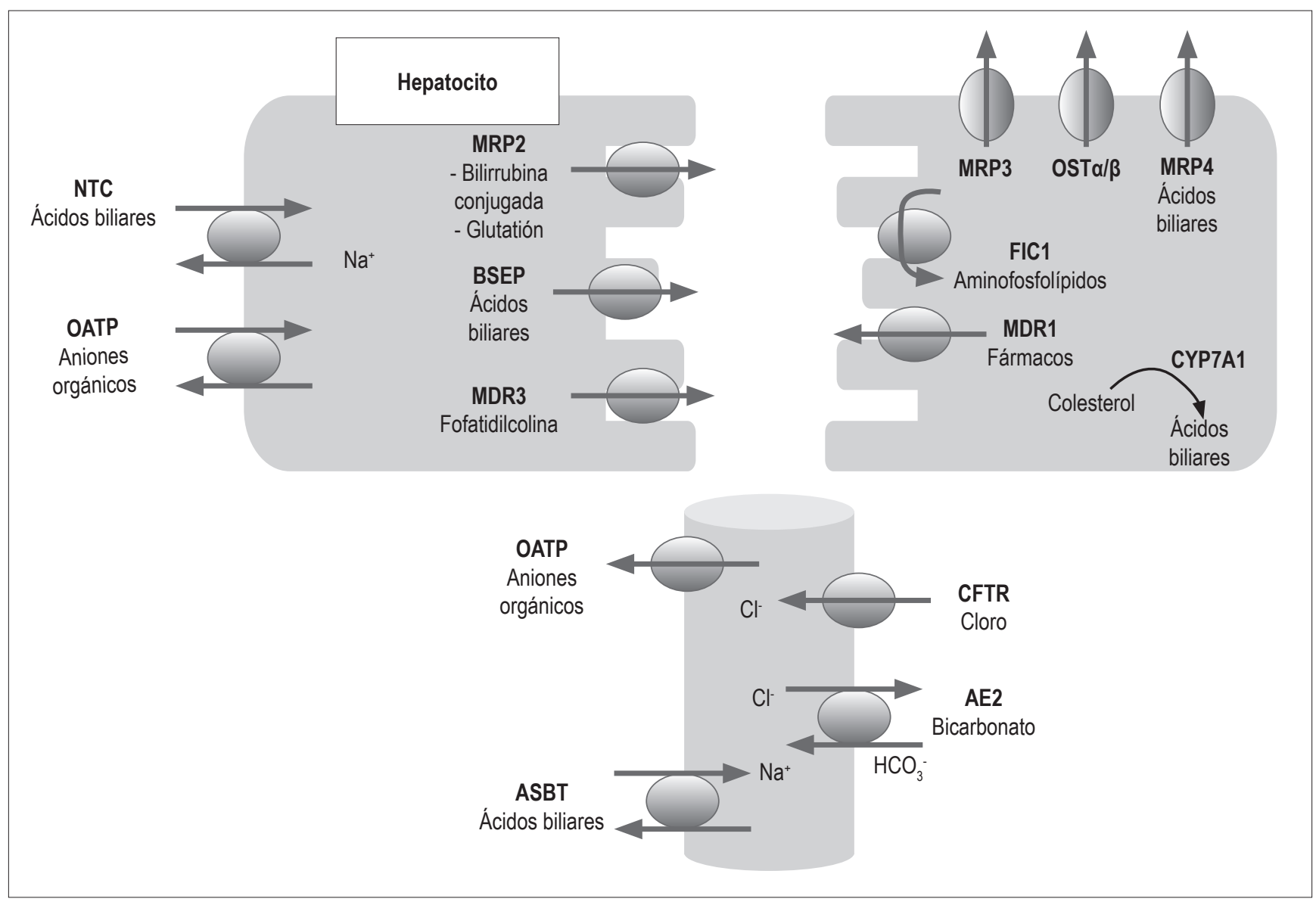

Figura 2. Transportadores biliares. Los ácidos biliares hepatocelulares provienen tanto del colesterol como de la síntesis de novo vía CYP7A1, que remplaza a la que se pierde diariamente en las heces (3\%-5\%), o vía captación hepatocelular desde la sangre sinusoidal de la circulación enterohepática. Esta se encuentra mediada por el cotransportador de alta afinidad $\mathrm{Na}^{+}$/taurocolato (NTCP) y la familia de transportadores de aniones orgánicos multiespecífica (OATP). La excreción canalicular de los componentes biliares vía transportadores dependientes de ATP determina la velocidad de la síntesis biliar. La membrana canalicular contiene una bomba exportadora de sales biliares para ácidos biliares monovalentes (BSEP); una bomba para exportar bilirrubina conjugada (MRP2) media la excreción de diferentes aniones orgánicos, como la bilirrubina y los ácidos biliares. La bomba exportadora de fosfolípidos (MDR3) saca fosfatidilcolina, la cual forma micelios mixtos junto con los ácidos biliares y el colesterol. Los fármacos catiónicos son excretados por la bomba exportadora de múltiples fármacos (MDR1). Adicionalmente la membrana canalicular contiene ATPasa de tipo P, FIC1, la cual es una flipasa de aminofosfolípidos. En la membrana basolateral, las bombas de ácidos biliares adicionales, MRP3, MRP4 y el transportador de heterodímeros orgánicos solubles OST $\alpha / \beta$ refuerzan la exportación sinusoidal de ácidos biliares. En condiciones normales, este sistema de transporte se expresa en niveles muy bajos, aunque puede ser inducido por condiciones colestásicas o medicamentos. Una isoforma 2 del intercambiador $\mathrm{Cl}^{-} /$ $\mathrm{HCO}_{3}^{-}$(AE2) media la excreción biliar de bicarbonato tanto en los hepatocitos como en los colangiocitos. El regulador de conductancia transmembranal de la fibrosis quística (CFTR) impulsa la excreción de bicarbonato por vía AE2 exclusivamente en los colangiocitos. El epitelio biliar también está involucrado en la reabsorción de ácidos biliares por medio del transportador apical $\mathrm{Na}^{+}$dependiente (ASBT) y su contraparte basolateral OST $\alpha / \beta$. Modificada de: Wagner M, et al. 2009.

nido y está críticamente involucrado en la disminución de la producción de ácidos biliares (CYP7A1) y de su absorción, tanto de forma $\mathrm{Na}^{+}$dependiente (NTCP) como $\mathrm{Na}^{+}$ independiente (OATP1B1 y OATP1B3); además, activa la excreción canalicular monovalente (BSEP) y divalente (MRP2 y MDR3) de ácidos biliares y de bilirrubina conjugada (MRP2) (figura 3) $(15-17,19)$.

\section{FISIOPATOLOGÍA Y MECANISMOS DE COLESTASIS}

La colestasis es la base del daño en los diferentes tipos de manifestaciones y patrones clínicos de DILI (20). Los fármacos pueden causar colestasis inhibiendo la expresión y la función de los transportadores hepatocelulares en la mayoría de los casos y, en raras ocasiones, induciendo el síndrome de 


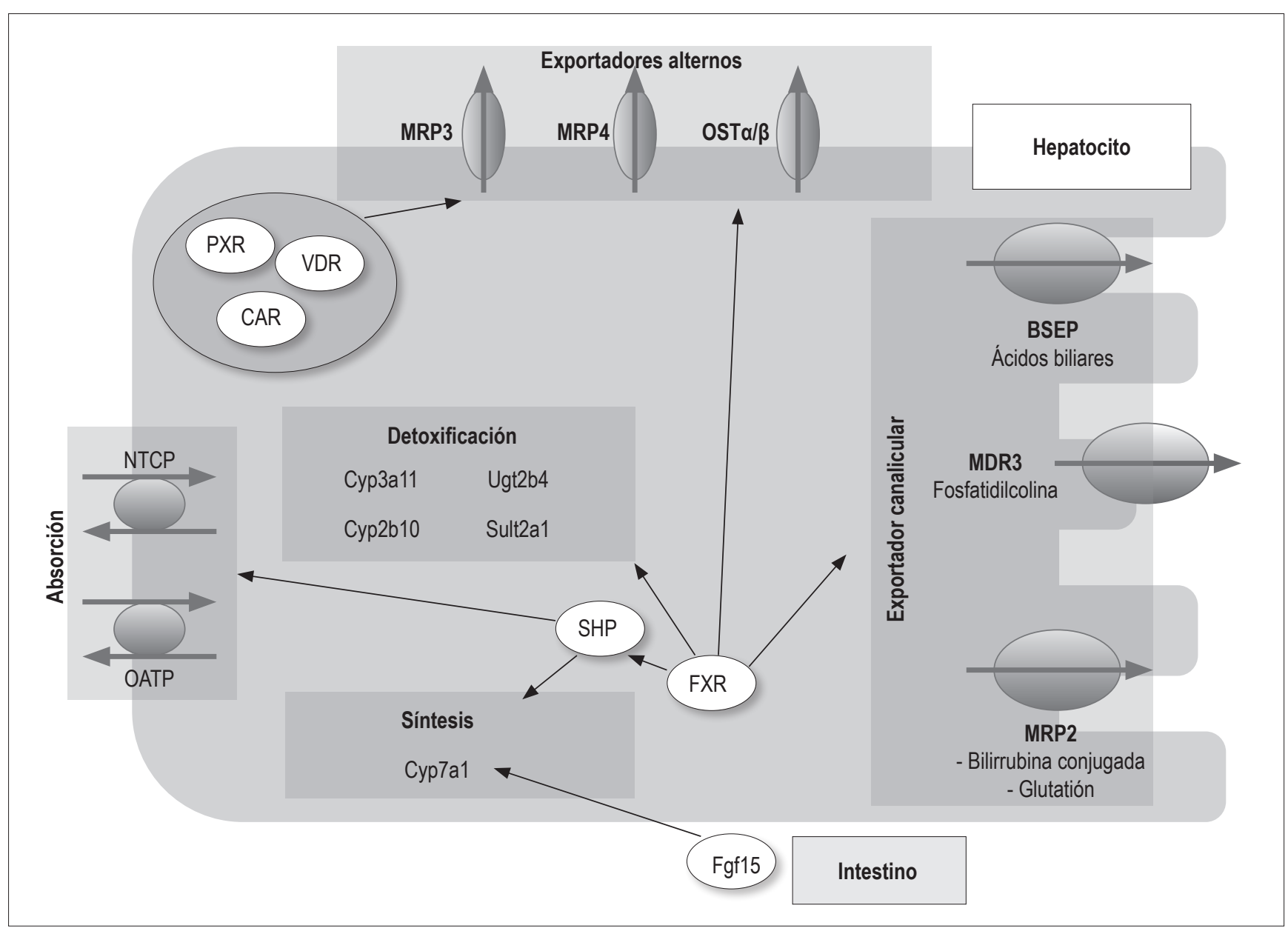

Figura 3. Principios generales de la regulación de las enzimas y transportadores hepatobiliares de ácidos biliares dependientes de los receptores nucleares (esquema de un modelo en roedores). Los principales procesos transcripcionales en humanos son similares aunque mucho menos estudiados. El principal receptor nuclear involucrado en la regulación de la formación biliar es el FXR activado por ácidos biliares. Este regula el metabolismo de los ácidos biliares tanto directamente (como estimulador del exportador canalicular de ácidos biliares vía BSEP y MRP2, y del exportador de fosfolípidos vía MDR2), como indirectamente (inhibiendo vía SHP los transportadores basolaterales $\mathrm{Na}^{+}$dependientes $[\mathrm{NTCP}]$, los $\mathrm{Na}^{+}$independientes [OATP] y la absorción y síntesis de ácidos biliares vía Cyp7a1). Adicionalmente, Fgf15, derivado de los enterocitos ileales, regula fuertemente a la baja la transcripción de Cyp7a1 y, por tanto, representa un sensor intestinal para el requerimiento de ácidos biliares. A excepción de OST $\alpha / \beta$, que también es regulado por FXR, los sistemas de exportadores alternos funcionan de forma independiente de este gen. PXR y VDR regulan positivamente MRP3; mientras que CAR regula positivamente tanto MRP3 como MRP4. El metabolismo de fase I (Cyp3a11 y Cyp2b10) y de fase II (Sult2a1) es estimulado por CAR y PXR; sin embargo, FXR es capaz de estimular ladetoxificación de fase I vía Cyp3a11 y la glucuronidación y sulfatación de fase II vía Ugt2b4 y Sult2a1. Modificada de: Wagner M, et al. 2009.

desaparición de conductos biliares, el cual puede progresar a cirrosis biliar (21). Muchos casos de colestasis inducida por fármacos resultan de la inhibición funcional que hace el medicamento o sus metabolitos de diferentes proteínas de transporte, y por lo tanto de sus vías; la principalmente afectada, y aún más en DILI colestásica, es la exportación de ácidos biliares dependiente de ATP vía BSEP (22) ABCB11, inhibida de forma directa y competitiva por medicamentos como rifampicina, ciclosporina, troglitazona y glibenclamida, e indirecta por metabolitos de hormonas esteroideas como estrógenos y progesterona (23-26).
También las alteraciones genéticas en la familia de transportadores ligadores de ATP se han asociado con desórdenes colestásicos que van desde la colestasis intrahepática familiar progresiva y la colestasis intrahepática recurrente benigna, hasta la colestasis intrahepática del embarazo, la colestasis inducida por medicamentos o drogas, la colelitiasis intrahepática y la cirrosis biliar.

Las alteraciones homocigóticas ocasionan variantes de gran impacto que dan lugar a síndromes colestásicos a temprana edad (29-32). Los defectos heterocigóticos en los transportadores predisponen la adquisición de colestasis 
por medio del reto con medicamentos, drogas, hormonas o inflamación, que causan descompensación de los defectos leves a moderados (32).

\section{CARACTERÍSTICAS CLÍNICAS, BIOQUÍMICAS E HISTOPATOLÓGICAS}

\section{Clínica}

La colestasis inducida por fármacos puede clasificarse teniendo en cuenta el sitio anatómico y el patrón bioquímico e histopatológico de la lesión, que puede ir desde una alteración hepática que interfiere con la síntesis de bilis hasta una alteración de los conductos que permiten su excreción $(33,34)$. Usualmente, este tipo de colestasis es una enfermedad aguda que remite rápidamente una vez se suspende el agente provocador (33). Se caracteriza clínicamente por ictericia, prurito, anorexia, malestar, náuseas y fatiga; además, puede incluir otras manifestaciones dependiendo del mecanismo causal de la lesión y de la toxicidad extrahepática del medicamento (35). En las formas de hipersensibilidad se pueden ver manifestaciones sistémicas como fiebre, erupción o eosinofilia. Es importante considerar que esta patología puede cursar con un espectro que va desde anormalidades bioquímicas asintomáticas hasta la insuficiencia hepática aguda $(33,34)$.

Algunos medicamentos pueden llevar a colestasis crónica con características similares a la cirrosis biliar primaria (CBP), con xantomas, prurito y melanodermia (35); estas formas se consideran benignas, ya que rara vez son progresivas y se pueden diferenciar de la CBP por la ausencia de anticuerpos antimicrosomales, el inicio agudo de los síntomas, las características histopatológicas y la presentación clínica atípica (33).

\section{Bioquímica}

$\mathrm{Al}$ igual que en otras formas colestásicas de lesión hepática, la valoración bioquímica muestra principalmente elevaciones en la fosfatasa alcalina (ALP) y aumento en la gamma glutamil transpeptidasa (GGT) (33). La aspartato aminotransferasa (AST) y la alanino aminotransferasa (ALT) pueden encontrarse en niveles normales o ligeramente elevados, y aunque las concentraciones de bilirrubina usualmente se encuentran elevadas, su valor dependerá del mecanismo de lesión del medicamento (35).

\section{Histopatología}

Histológicamente, la colestasis inducida por fármacos puede presentarse como una lesión aguda o crónica, com- prometiendo o no el parénquima hepático (35). Las formas agudas son las más comunes y se clasifican en:

- Colestasis pura o blanda: se encuentran coágulos de pigmento biliar en los canalículos, que aparecen distendidos, y acumulación de pigmento en los hepatocitos y las células de Kupffer, en ausencia de inflamación o lesión hepatocelular; esto es más prominente en la zona 3 o zona centrolobulillar $(35,36)$. Hacen parte de este grupo los medicamentos como esteroides anabólicos, anticonceptivos orales y warfarina $(34,37)$.

- Hepatitis colestásica (colangitis o colestasis por hipersensibilidad): se observa compromiso hepatocelular, que se manifiesta también en la bioquímica hepática (35); se puede acompañar de proliferación de conductos y pueden encontrarse neutrófilos, linfocitos y eosinófilos en caso de colangitis (36). Este patrón es común en la toxicidad por eritromicina o por clorpromazina $(34,35,37)$.

En las formas crónicas de la enfermedad (más de 6 meses de duración) se observan cambios pseudoxantomatosos con apariencia espumosa debido al acúmulo de ácidos biliares en los hepatocitos, especialmente en las regiones periportales (33); además, puede demostrarse el contenido elevado de cobre con tinciones especiales y, ocasionalmente, evidenciarse la presencia de cuerpos de Mallory (37). La colestasis crónica puede dividirse igualmente:

- Síndrome de desaparición de los conductos biliares: se presenta inicialmente como inflamación hepatocelular y del conducto biliar, llevando a ductopenia y en algunos casos a cirrosis al prolongarse la noxa. Es una de las presentaciones más severas y puede desencadenarse por carbamazepina, clorpromazina, ibuprofeno, amoxicilina y clindamicina. Debe hacerse diagnóstico diferencial con CBP y enfermedad obstructiva $(35,49)$.

- Esclerosis biliar: puede darse cuando se presenta una lesión isquémica de los conductos intrahepáticos y extrahepáticos, simulando radiológica e histológicamente a la colangitis esclerosante primaria $(35,36)$. Los medicamentos asociados con esta presentación incluyen la 5-fluorodeoxiuridina (para el tratamiento de metástasis hepáticas del cáncer colorrectal) y el formaldehido $(35,36)$.

El edema del área portal, los infartos y los lagos biliares son expresiones tardías de obstrucción mecánica de los conductos y rara vez se manifiestan en la colestasis por fármacos (33); sin embargo, la presentación histopatológica temprana de la obstrucción biliar es indistinguible de la colestasis inducida por medicamentos, siendo entonces un diagnóstico diferencial importante (35-38). 
En la tabla 1 se puede encontrar un resumen de las características bioquímicas e histopatológicas más comunes de los patrones colestásicos descritos, y en la tabla 2 se encuentran los medicamentos más asociados con el patrón colestásico de toxicidad hepática.

\section{FACTORES DE RIESGO}

Entre los factores de riesgo para DILI se encuentra la edad, explicado posiblemente por cambios en la expresión de receptores y transportadores, en el porcentaje de grasa corporal, en los volúmenes de distribución y en el estado hormonal $(7,36)$. El género, aunque no representa una diferencia significativa en su incidencia, sí ha sido relacionado con cambios en la presentación y el pronóstico, siendo en los hombres más común el patrón colestásico y teniendo las mujeres un curso menos favorable de la enfermedad (39). También han sido identificados factores genéticos y polimorfismos para el metabolismo farmacológico como el antígeno leucocitario humano (HLA) B*5701, los haplotipos-DRB y-DRBQ y el polimorfismo MDR3/BSEP (que se asocia con predisposición para colestasis en el embarazo e inducida por esteroides) $(36,40,50)$. Igualmente, se han descrito como factores de riesgo los antecedentes de consumo de alcohol, de enfermedad hepática (incluyendo esteatohepatitis) y de infección por VIH (por un mecanismo aún desconocido) (7).

Adicionalmente, se han relacionado con mayor fuerza factores dependientes del medicamento como la composición, la dosis y el metabolismo; por ejemplo los esteroides con sustituciones en C-17 (particularmente alquilación o metilación) (36), las dosis mayores de $50 \mathrm{mg} /$ día y el uso concomitante de varios medicamentos de metabolismo hepático $(36,39,41)$.

\section{DIAGNÓSTICO}

No existe un examen o marcador que sea indicador absoluto de DILI, por lo que es principalmente un diagnóstico de exclusión basado en evidencia circunstancial. El abordaje inicial de su sospecha debe abarcar una historia clínica detallada que incluya un interrogatorio exhaustivo acerca de los antecedentes médicos y factores de riesgo, uso de fármacos formulados o automedicados, y de sustancias no convencionales como medicina alternativa y herbales; adicionalmente, se debe indagar sobre el consumo de alcohol $\mathrm{y}$ otras sustancias psicoactivas. Muchas veces este interrogatorio incluye no solo al paciente, sino también a su fami-

Tabla 1. Tipos de colestasis inducida por fármacos. En la tabla se encuentran las características histológicas y bioquímicas que permiten identificar y diferenciar los patrones colestásicos de DILI. Modificada de: Mohi-ud-din R, et al. 2004.

\begin{tabular}{|c|c|c|c|c|c|}
\hline \multicolumn{6}{|c|}{ Término morfológico (término clínico) } \\
\hline & $\begin{array}{c}\text { Patrón } \\
\text { canalicular } \\
\text { (ictericia blanda) }\end{array}$ & $\begin{array}{c}\text { Patrón } \\
\text { hepatocanalicular } \\
\text { (hepatitis colestásica) }\end{array}$ & $\begin{array}{l}\text { Patrón ductular } \\
\text { (colangiolar) }\end{array}$ & $\begin{array}{c}\text { Patrón colangio-destructivo } \\
\text { (síndrome de conductillos } \\
\text { evanescentes) }\end{array}$ & $\begin{array}{l}\text { Patrón de colangitis } \\
\text { esclerosante septal } \\
\text { (colangioesclerosis) }\end{array}$ \\
\hline \multicolumn{6}{|c|}{ Características histológicas } \\
\hline Pigmento biliar & + & + & +++ & + & + \\
\hline Inflamación portal & 0 & $++^{\circ}$ & $+/-$ & + & + \\
\hline Necrosis hepatocelular & - & + & $+/-$ & + & + \\
\hline Destrucción ductular & 0 & + & $+/-$ & +++ & +++ \\
\hline Colangitis & - & $+/-$ & + & + & + \\
\hline Peliosis & + & + & - & - & - \\
\hline Hipersensibilidad & No & Frecuente & No & No & No \\
\hline \multicolumn{6}{|l|}{ Características bioquímicas } \\
\hline Bilirrubina & +++ & +++ & +++ & $+/+++$ & $+/+++^{a}$ \\
\hline Fosfatasa alcalina & $<3$ veces $L S N$ & 3-10 veces LSN & $>3$ veces $L S N$ & $>3$ veces LSN & $>3$ veces $L S N$ \\
\hline AST/ALT & $1-8$ veces LSN & 2-10 veces LSN & $2-10$ veces LSN & 2-10 veces LSN & $2-10$ veces LSN \\
\hline Colesterol & $+/-$ & ++ & $+/-$ & +++ & +++ \\
\hline Ejemplo & $\begin{array}{l}\text { ACO, esteroides } \\
\text { anabólicos }\end{array}$ & $\begin{array}{l}\text { Clorpromazina, } \\
\text { eritromicina }\end{array}$ & Benaxaprofeno & Paraquat, clorpromazina & $\begin{array}{c}\text { Infusión de fluxuridina, } \\
\text { escolicidas }\end{array}$ \\
\hline
\end{tabular}

${ }^{\circ}$ : usualmente temprana.

a: usualmente a los 3-6 meses. 
Tabla 2. Medicamentos asociados con lesión colestásica. Modificada de: Mohi-ud-din R, et al. 2004.

\begin{tabular}{|c|c|c|}
\hline \multicolumn{3}{|c|}{ Medicamentos asociados a lesión colestásica } \\
\hline Antimicrobianos & Antiinflamatorios & Estatinas \\
\hline Antifúngicos & Benoxaprofeno & Pravastatina \\
\hline Griseofulvina & Celecoxib & Esteroides y sus inhibidores \\
\hline Itraconazol & Diclofenaco & Esteroides anabólicos \\
\hline Ketoconazol & Ibuprofeno & Anticonceptivos orales \\
\hline Terbinafina & Sales de oro & Danazol \\
\hline Tiabendazol & Indometacina & Estradiol \\
\hline Cefalosporinas & Infliximab & Tamoxifeno \\
\hline Macrólidos & Meloxicam & Otros \\
\hline Azitromicina & Piroxicam & Alopurinol \\
\hline Claritromicina & Anticonvulsivantes & Beta caroteno \\
\hline Eritromicina & Carbamazepina & Dapsona \\
\hline Quinolonas & Agentes cardiovasculares & Fluorodesoxiuridina \\
\hline Ciprofloxacina & IECA & HAART \\
\hline Norfloxacina & Captopril & Remedios herbales \\
\hline Penicilinas & Fosinopril & Mesalamina \\
\hline Amoxicilina-clavulanato & ARA-II & Propafenona \\
\hline Dicloxacilina & Irbesartran & Tacrolimús \\
\hline Floxacilina & Simpaticolíticos de acción central & Terfenadina \\
\hline Oxacilina & Metildopa & Nutrición parenteral total \\
\hline Otros & Diuréticos & \\
\hline Nitrofurantoína & Tiazidas & \\
\hline Tetraciclinas & Anticoagulantes y antiagregantes & \\
\hline Trimetroprim-sulfametoxazol & Heparina & \\
\hline Rifampicina & Clopidrogrel & \\
\hline Psicotrópicos & Warfarina & \\
\hline Antipsicóticos & Inmunosupresores & \\
\hline Clorpromazina & Azatioprina & \\
\hline Haloperidol & Ciclofosfamida & \\
\hline Risperidona & 6-Mercaptopurina. & \\
\hline Sedantes & Antagonistas del receptor $\mathrm{H}_{2}$ & \\
\hline Barbitúricos & Cimetidina & \\
\hline Antidepresivos tricíclicos & Ranitidina & \\
\hline Imipramina & Hipoglucemiantes & \\
\hline Amitriptilina & Glimepiride & \\
\hline ISRS & Metformina & \\
\hline Sertralina & Pioglitazona & \\
\hline
\end{tabular}


lia. Todos estos datos deben ir acompañados del tiempo de inicio del consumo para asociarlo temporalmente con la lesión hepática, sobre todo en los pacientes polimedicados.

Lo anterior se debe complementar con los exámenes bioquímicos hepáticos usuales, las pruebas de coagulación estándar, los marcadores serológicos y las imágenes biliares de rutina para descartar otras causas más comunes de colestasis, las cuales se pueden evidenciar en la tabla $3(7,36$, 42). Otros exámenes en busca de enfermedades autoinmunes o infecciosas pueden ser útiles para explorar los diagnósticos diferenciales; aunque no siempre es necesaria, la biopsia hepática puede ser esencial en casos seleccionados, especialmente para valorar el pronóstico (36).

Existen situaciones más sugestivas de DILI, como el inicio de un nuevo medicamento en los últimos 3 meses, la presencia de erupción o eosinofilia, la enfermedad hepática de tipo mixto (compromiso hepatocelular y colestásico), la colestasis sin alteraciones en la imagenología, la hepatitis aguda sobre crónica sin autoanticuerpos o hipergammaglobulinemia y los pacientes con factores de riesgo. La ausencia de lo anterior no descarta la posibilidad de tener colestasis inducida por fármacos, o cualquier otra forma de DILI, pero tenerlos en cuenta puede facilitar un diagnóstico más temprano (7).

Se han desarrollado varias escalas que permiten valorar la probabilidad de DILI. Entre ellas, la más ampliamente usada y validada es la CIOMS/RUCAM propuesta por Danan y Benichou en la Reunión de Consenso Internacional en 1990 (8); esta permite clasificar el diagnóstico de DILI en definitivo, probable, posible, poco probable y descartado, según el patrón de lesión hepática (hepatocelular o colestásica/ mixta), los criterios cronológicos, el curso de la enfermedad, los factores de riesgo, la información disponible sobre hepatotoxicidad del medicamento, la exclusión de otras causas y la respuesta a la readministración del medicamento; este último criterio no se debe tener en cuenta por los riesgos que conlleva para el paciente. En la tabla 4 se muestra esta escala de probabilidad de DILI para el patrón colestásico.

Otros exámenes adicionales útiles para el diagnóstico incluyen la prueba de estimulación linfocitaria con medicamento (Drug-lynphocyte stimulation test - DLST) y la prueba de migración leucocitaria (leukocite migration test-LMT). La primera tiene una sensibilidad apenas del $50 \%$ y no permite relacionar inequívocamente el fármaco con el daño hepático (43); mientras que la LMT ha probado ser más útil para la identificación de la sustancia agresora (44). Estas y otras pruebas de este tipo son útiles para identificar el medicamento específico que produjo el daño, pero son complejas, costosas y no se recomiendan para uso rutinario (7).
Tabla 3. Exámenes diagnósticos iniciales en el abordaje de DILI. En la tabla se encuentran enlistados los exámenes que pueden ser de utilidad en la valoración de DILI, la marcación * indica aquellos que deben hacerse inicialmente. Modificada de: Tajiri, et al. 2008.

\begin{tabular}{|c|c|}
\hline \multicolumn{2}{|c|}{ Exámenes diagnósticos iniciales en el abordaje de DILI } \\
\hline Prueba & Utilidad clínica \\
\hline \multicolumn{2}{|l|}{ Hematológicos* } \\
\hline Hemograma (incluidos eosinófilos) & $\begin{array}{l}\text { Reacciones de } \\
\text { hipersensibilidad }\end{array}$ \\
\hline \multicolumn{2}{|l|}{ Bioquímicos* } \\
\hline $\begin{array}{l}\text { AST/ALT } \\
\text { Lactato deshidrogenasa } \\
\text { Gammaglutamil transpeptidasa } \\
\text { Fosfatasa alcalina }\end{array}$ & $\begin{array}{l}\text { Definición del patrón de daño } \\
\text { hepático }\end{array}$ \\
\hline $\begin{array}{l}\text { Bilirrubina total y directa. } \\
\text { Albúmina }\end{array}$ & Gravedad de la lesión \\
\hline \multicolumn{2}{|l|}{ Coagulación* } \\
\hline $\begin{array}{l}\text { Protrombina } \\
\text { INR }\end{array}$ & Gravedad de la lesión \\
\hline \multicolumn{2}{|l|}{ Serológicos (autoinmunidad)* } \\
\hline $\begin{array}{l}\lg G, \lg A, \lg M \\
\text { Anticuerpos anti-nucleares (ANA) } \\
\text { Anticuerpos anti-mitocondriales } \\
\text { (AMA) }\end{array}$ & $\begin{array}{l}\text { Diagnóstico diferencial } \\
\text { (solicitar de acuerdo con la } \\
\text { sospecha clínica) }\end{array}$ \\
\hline \multicolumn{2}{|l|}{ Serología viral } \\
\hline $\begin{array}{l}\text { IgM anti-HA* } \\
\text { HBsAg*, IgM-HBc*, anti-HBc, HBV- } \\
\text { DNA } \\
\text { Anti-HCV*, HCV-RNA } \\
\text { Anti-HDV, HDV-DNA } \\
\text { Anti-HEV, HEV-RNA } \\
\text { lgM-EBV } \\
\text { lgM-CMV }\end{array}$ & $\begin{array}{l}\text { Diagnóstico diferencial } \\
\text { (solicitar de acuerdo con la } \\
\text { sospecha clínica) }\end{array}$ \\
\hline \multicolumn{2}{|l|}{ Imágenes } \\
\hline Ecografía transabdominal & $\begin{array}{l}\text { Diagnóstico diferencial } \\
\text { (solicitar de acuerdo con la } \\
\text { sospecha clínica) }\end{array}$ \\
\hline
\end{tabular}

ALT: alanino aminotransferasa; AST: aspartato aminotransferasa; CMV: citomegalovirus; HA: hepatitis A; HBc: hepatitis B core; HBsAg: antígeno de superficie de hepatitis B; IgA: inmunoglobulina A; IgG: inmunoglobulina G; IgM: inmunoglobulina M; VEB: virus de EpsteinBarr; VHC: virus de la hepatitis C; VHD: virus de la hepatitis D; VHE: virus de la hepatitis $\mathrm{E}$.

\section{TRATAMIENTO}

En la mayoría de los casos, se espera que el cuadro mejore con el retiro del medicamento responsable del daño; sin embargo, se ha visto que algunos pacientes mejoran incluso 
Tabla 4. Puntaje CIOMS/RUCAM para la valoración de DILI. Se interpreta el resultado así: mayor de 8: definitivo; 6-8: probable; 3-5 posible; 1-2: poco probable; menor o igual a 0: descartado. Modificada de: Danan G, et al. 2014.

\begin{tabular}{|c|c|c|c|c|c|c|}
\hline \multicolumn{7}{|c|}{ CIOMS/RUCAM } \\
\hline \multirow{2}{*}{$\begin{array}{l}\text { Tipo de lesión hepática } \\
\text { Criterios cronológicos }\end{array}$} & \multicolumn{2}{|c|}{ Hepatocelular } & \multirow[t]{2}{*}{ Valor } & \multicolumn{2}{|c|}{ Colestásico/mixto } & \multirow[t]{2}{*}{ Valor } \\
\hline & Primera exposición & Segunda exposición & & Primera exposición & Segunda exposición & \\
\hline \multirow{2}{*}{$\begin{array}{l}\text { Tiempo de la ingesta del } \\
\text { medicamento al inicio de } \\
\text { los síntomas }\end{array}$} & 5-90 días & 1-15 días & 2 & 5-90 días & 1-90 días & 2 \\
\hline & $<50>90$ días & $>15$ días & 1 & $<50>90$ días & $>90$ días & 1 \\
\hline $\begin{array}{l}\text { Tiempo de retiro del } \\
\text { medicamento al inicio de } \\
\text { los síntomas }\end{array}$ & $<15$ días & $\geq 15$ días & 1 & $\leq 30$ días & $\leq 30$ días & 1 \\
\hline Curso de la enfermedad & \multicolumn{2}{|c|}{$\begin{array}{c}\text { Diferencia entre valor máximo de ALT y límite } \\
\text { superior normal }\end{array}$} & \multicolumn{4}{|c|}{$\begin{array}{l}\text { Diferencia entre valor máximo de ALP y límite } \\
\text { superior normal }\end{array}$} \\
\hline \multirow[t]{4}{*}{ Al retiro del medicamento } & \multicolumn{2}{|l|}{ Mejoría $>50 \%$ en 8 días } & 3 & \multicolumn{2}{|c|}{ Mejoría $>50 \%$ en 180 días } & 2 \\
\hline & \multicolumn{2}{|l|}{ Mejoría $>50 \%$ en 30 días } & 2 & \multicolumn{2}{|l|}{ Mejoría $<50 \%$ en 180 días } & 1 \\
\hline & \multicolumn{2}{|c|}{ Falta información o no hay mejoría } & 0 & \multicolumn{2}{|c|}{ Falta información o no hay mejoría } & 0 \\
\hline & \multicolumn{2}{|c|}{ Empeoramiento o mejoría $<50 \%$ en 30 días } & -1 & & & \\
\hline \multirow[t]{2}{*}{ Factores de riesgo } & \multicolumn{2}{|l|}{ Edad ( $\geq 55$ años) } & 1 & \multicolumn{2}{|l|}{ Edad ( $\geq 55$ años) } & 1 \\
\hline & Consumo de alcohol & & 1 & \multicolumn{2}{|c|}{ Consumo de alcohol o embarazo } & 1 \\
\hline \multirow[t]{5}{*}{ Terapia concomitante } & \multicolumn{2}{|l|}{ Ninguno o se desconoce } & 0 & \multicolumn{2}{|c|}{ Ninguno o se desconoce } & 0 \\
\hline & \multicolumn{2}{|c|}{ Fármaco con contribución sugestiva } & -1 & \multicolumn{2}{|c|}{ Fármaco con contribución sugestiva } & -1 \\
\hline & \multicolumn{2}{|c|}{ Hepatotoxina conocida, contribución sugestiva } & -2 & \multicolumn{2}{|c|}{ Hepatotoxina conocida, contribución sugestiva } & -2 \\
\hline & \multicolumn{2}{|c|}{ Rol probado en el caso } & -3 & \multicolumn{2}{|l|}{ Rol probado en el caso } & -3 \\
\hline & No hay información disp & onible & 0 & No hay información disp & onible & 0 \\
\hline Exclusión de otras causas & Descartada & & 2 & Descartada & & 2 \\
\hline no medicamentosas & Posible a no investigad & & -2 a 1 & Posible a no investigad & & -2 a 1 \\
\hline & Otra causa probable & & -3 & Otra causa probable & & -3 \\
\hline Información de & Reacción desconocida & & 0 & Reacción desconocida & & 0 \\
\hline hepatotoxicidad previa & Publicada pero no etiqu & tada en el medicamento & 1 & Publicada pero no etiqu & tada en el medicamento & 1 \\
\hline & Etiquetada en las carac & erísticas del medicamento & 2 & Etiquetada en las carac & erísticas del medicamento & 2 \\
\hline Respuesta a & Positiva & & 3 & Positiva & & 3 \\
\hline readministración del & Compatible & & 1 & Compatible & & 1 \\
\hline medicamento & Negativa & & -2 & Negativa & & -2 \\
\hline & No disponible o no inter & retable & 0 & No disponible o no inter & retable & 0 \\
\hline & $\begin{array}{l}\text { Concentraciones en pla } \\
\text { tóxicas }\end{array}$ & ma conocidas como & 3 & $\begin{array}{l}\text { Concentraciones en pla } \\
\text { tóxicas }\end{array}$ & ma conocidas como & 3 \\
\hline Pruebas de laboratorio & Positivos & & 3 & Positivos & & 3 \\
\hline validadas con buenos & Negativos & & -3 & Negativos & & -3 \\
\hline valores predictlvos & No disponibles o no inte & pretables & 0 & No disponibles o no inte & pretables & 0 \\
\hline
\end{tabular}

sin su suspensión, por lo que es necesario correlacionar la gravedad del cuadro clínico con la importancia del uso del medicamento (7).

Aunque no existen criterios definitivos para la suspensión del fármaco, se han propuesto los siguientes ante la sospecha de DILI colestásica $(7,45)$ por su asociación con daño hepático:
- Elevación de la bilirrubina $>3$ veces el límite superior normal

- $\operatorname{INR}>1,5$

Además, en cualquier tipo de patrón de DILI, son consideraciones para retiro del medicamento (46):

- ALT o AST >8 veces el límite superior normal 
- ALT o AST >5 veces el límite superior normal por más de 2 semanas

- ALT o AST >3 veces el límite superior normal con aumento de la bilirrubina total $>2$ veces el límite superior normal o con INR $>1,5$

- ALT o AST >3 veces el límite superior normal con fatiga, náuseas, vómito, dolor abdominal en cuadrante superior derecho, fiebre, erupción y/o eosinofilia $>5 \%$
El uso de otros medicamentos para el manejo de DILI colestásica no tiene apoyo en la literatura, y aunque se ha utilizado el tratamiento con UDCA (ácido ursodesoxicólico) y con corticoesteroides (en pacientes con sospecha de hipersensibilidad), los ensayos clínicos controlados no han probado su eficacia $(7,46,47,51)$. La hemodiálisis rara vez está indicada, y siempre que haya algún indicio de insuficiencia hepática aguda, el paciente debe ser hospitalizado y

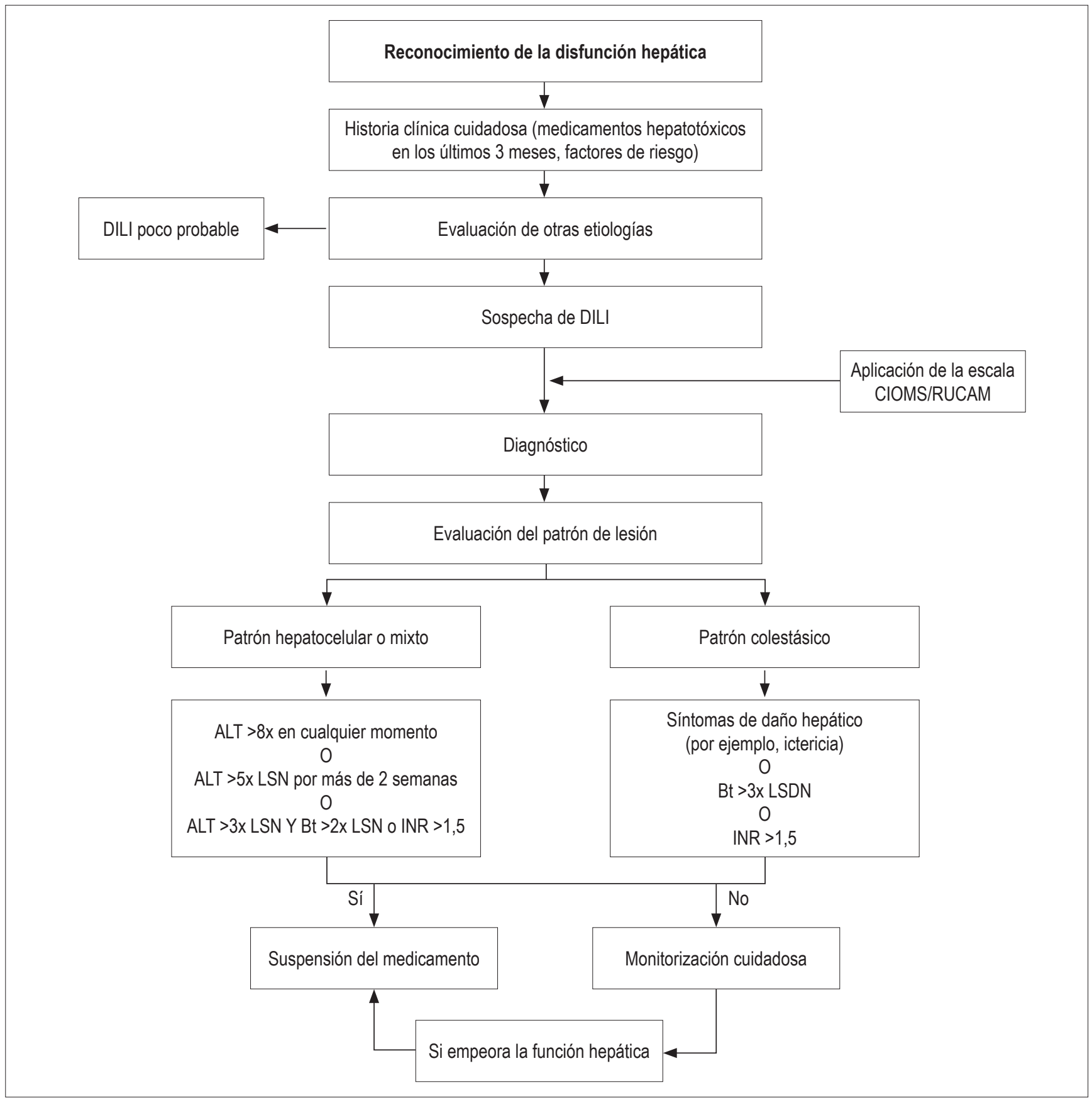

Figura 4. Algoritmo para el abordaje de DILI. Bt: bilirrubina total; LSN: límite superior normal. Modificada de: Tajiri, et al. 2008. 
considerado para trasplante hepático $(46,47)$. En la figura 4 se describe una propuesta de algoritmo para el diagnóstico y manejo de DILI colestásica.

\section{PRONÓSTICO}

La información sobre el desenlace de la presentación colestásica y mixta es limitada. Antes se creía que el patrón colestásico de DILI se asociaba con un mejor pronóstico de la enfermedad; sin embargo, la literatura reciente muestra que entre el 5\% al 13\% de estos pacientes desarrollan enfermedad hepática crónica (más frecuente que en aquellos con patrón hepatocelular), y que entre el 5\% al 14\% mueren o requieren trasplante por esta causa $(36,48)$.

El pronóstico a largo plazo para DILI, en general, depende de la presentación clínica y bioquímica inicial del paciente, siendo las aminotransferasas y la bilirrubina total los mayores predictores de mortalidad. Aquellos que debutan con elevación de las aminotransferasas (ALT mayor o igual de 3 veces el límite superior normal) e ictericia (bilirrubina total 2 o más veces el límite superior normal) tienen una mortalidad de entre el $10 \%$ al $50 \%$, esto es lo que se conoce como Ley de Hy $(48,52)$.

\section{Agradecimientos}

Agradecemos algrupo de gastrohepatología de la Universidad de Antioquia.

\section{Fuentes de financiación}

Los autores declaran no tener ninguna fuente de financiación para la realización del estudio.

\section{REFERENCIAS}

1. Vuppalanchi R, Liangpunsakul S, Chalasani N. Etiology of new-onset jaundice: How often is it caused by idiosyncratic drug-induced liver injury in the United States? Am J Gastroenterol. 2007;102(3):558-62.

2. Sgro C, Clinard F, Ouazir K, Chanay H, Allard C, Guilleminet $\mathrm{C}$, et al. Incidence of drug-induced hepatic injuries: A French population-based study. Hepatology. 2002;36(2):451-5.

3. Meier Y, Cavallaro M, Roos M, Pauli-Magnus C, Folkers G, Meier $\mathrm{P}$, et al. Incidence of drug-induced liver injury in medical inpatients. Eur J Clin Pharmacol. 2005;61(2):135-43.

4. Ostapowicz G, Fontana RJ, Schiødt F V, Larson A, Davern TJ, Han SHB, et al. Results of a Prospective Study of Acute Liver Failure at 17 Tertiary Care Centers in the United States. Ann Intern Med. 2002;137(12):947-54.

5. Fontana RJ, Hayashi PH, Gu J, Reddy KR, Barnhart H, Watkins $\mathrm{PB}$, et al. Idiosyncratic drug-induced liver injury is associated with substantial morbidity and mortality within 6 months from onset. Gastroenterology. 2014;147(1):96-108.

6. Fontana RJ, Seeff LB, Andrade RJ, Björnsson E, Day CP, Serrano J, et al. Standardization of nomenclature and causality assessment in drug-induced liver injury: Summary of a clinical research workshop. Hepatology. 2010;52(2):730-42.

7. Tajiri K. Practical guidelines for diagnosis and early management of drug-induced liver injury. World J Gastroenterol. 2008;14(44):6774-6785.

8. Danan G, Benichou C. Causality assessment of adverse reactions to drugs - I. A novel method based on the conclusions of international consensus meetings: Application to drug-induced liver injuries. J Clin Epidemiol. 2014;46(11):1323-30.

9. Bénichou C. Criteria of drug-induced liver disorders. Report of an international consensus meeting. J Hepatol. 1990;11(2):272-6.

10. Bhamidimarri KR, Schiff E. Drug-induced cholestasis. Clin Liver Dis. 2013;17(4):519-31, vii.

11. Andrade RJ, Lucena MI, Kaplowitz N, García-Munoz B, Borraz Y, Pachkoria K, et al. Outcome of acute idiosyncratic drug-induced liver injury: Long-term follow-up in a hepatotoxicity registry. Hepatology. 2006;44(6):1581-8.

12. Trauner M, Meier P, Boyer J. Molecular Pathogenesis of Cholestasis. N Engl J Med. 1998;339(17)1217-27.

13. Trauner M, Boyer JL. Bile Salt Transporters: Molecular characterization, function, and regulation. Physiol Rev. 2003;83(2):633-71.

14. Hofmann AF. The Continuing Importance of Bile Acids in Liver and Intestinal Disease. Arch Intern Med. 1999; 159(22):2647-2658.

15. Wagner M, Zollner G, Trauner M. New molecular insights into the mechanisms of cholestasis. J Hepatol. 2009;51(3):565-80.

16. Suchy FJ, Ananthanarayanan M. Bile salt excretory pump: Biology and pathobiology. J Pediatr Gastroenterol Nutr. 2006;43 Suppl 1:S10-6.

17. Goodwin B, Jones SA, Price RR, Watson MA, McKee DD, Moore LB, et al. A Regulatory cascade of the nuclear receptors FXR, SHP-1, and LRH-1 represses bile acid biosynthesis. Mol Cell. 2000;6(3):517-26.

18. Denson LA, Sturm E, Echevarria W, Zimmerman TL, Makishima M, Mangelsdorf DJ, et al. The orphan nuclear receptor, shp, mediates bile acid-induced inhibition of the rat bile acid transporter, ntcp. Gastroenterology. $2001 ; 121(1): 140-7$.

19. Lu TT, Makishima M, Repa JJ, Schoonjans K, Kerr TA, Auwerx J, et al. Molecular Basis for Feedback Regulation of Bile Acid Synthesis by Nuclear Receptors. Mol Cell. 2014;6(3):507-15.

20. Ogimura E, Sekine S, Horie T. Bile salt export pump inhibitors are associated with bile acid-dependent drug-induced toxicity in sandwich-cultured hepatocytes. Biochem Biophys Res Commun. 2011;416(3-4):313-7.

21. Pauli-Magnus C, Meier PJ. Hepatobiliary transporters and drug-induced cholestasis. Hepatology. 2006;44(4):778-87.

22. Lang C, Meier Y, Stieger B, Beuers U, Lang T, Kerb R, et al. Mutations and polymorphisms in the bile salt export 
pump and the multidrug resistance protein 3 associated with drug-induced liver injury. Pharmacogenet Genomics. 2007; 17(1):47-60.

23. Stieger B, Fattinger K, Madon J, Kullak-Ublick GA, Meier PJ. Drug- and estrogen-induced cholestasis through inhibition of the hepatocellular bile salt export pump (Bsep) of rat liver. Gastroenterology. 2000;118(2):422-30.

24. Fattinger $K$. The endothelin antagonist bosentan inhibits the canalicular bile salt export pump: A potential mechanism for hepatic adverse reactions. Clin Pharmacol Ther. 2001;69(4):223-31.

25. Funk C, Ponelle C, Scheuermann G, Pantze M. Cholestatic Potential of Troglitazone as a Possible Factor Contributing to Troglitazone-Induced Hepatotoxicity : In vivo and in vitro Interaction at the Canalicular Bile Salt Export Pump (Bsep) in the Rat. Mol Pharmacol. 2001;59(3):627-35.

26. Dawson S, Stahl S, Paul N, Barber J, Kenna JG. In vitro inhibition of the bile salt export pump correlates with risk of cholestatic drug-induced liver injury in humans. Drug Metab Dispos. 2012;40(1):130-8.

27. Smith AJ, van Helvoort A, van Meer G, Szabo K, Welker E, Szakacs G, et al. MDR3 P-glycoprotein, a phosphatidylcholine translocase, transports several cytotoxic drugs and directly interacts with drugs as judged by interference with nucleotide trapping. J Biol Chem. 2000;275(31):23530-9.

28. Bode KA, Donner MG, Leier I, Keppler D. Inhibition of transport across the hepatocyte canalicular membrane by the antibiotic fusidate. Biochem Pharmacol. 2002;64(1):151-8.

29. Sirvent A, Verhoeven AJM, Jansen H, Kosykh V, Darteil RJ, Hum DW, et al. Farnesoid X receptor represses hepatic lipase gene expression. J Lipid Res. 2004;45(11):2110-5.

30. OudeElferinkRPJ,Paulusma CC, GroenAK.Hepatocanalicular transport defects: pathophysiologic mechanisms of rare diseases. Gastroenterology. 2006;130(3):908-25.

31. Jacquemin E. Progressive familial intrahepatic cholestasis. Genetic basis and treatment. Clin Liver Dis. 2000;4(4):753-63.

32. Pauli-Magnus C, Stieger B, Meier Y, Kullak-Ublick G a, Meier PJ. Enterohepatic transport of bile salts and genetics of cholestasis. J Hepatol. 2005;43(2):342-57.

33. Mohi-ud-din R, Lewis JH. Drug- and chemical-induced cholestasis. Clin Liver Dis. 2004;8(1):95-132, vii.

34. Zimmerman HJ. Intrahepatic cholestasis. Arch Intern Med. 1979;139(9):1038-45.

35. Ramachandran R, Kakar S. Histological patterns in druginduced liver disease. J Clin Pathol. 2009;62(6):481-92.

36. Bhamidimarri KR, Schiff E. Drug-induced cholestasis. Clin Liver Dis. 2013;17(4):519-31, vii.

37. Green RM, Crawford JM. Hepatocellular cholestasis: Pathobiology and histological outcome. Semin Liver Dis. $1995 ; 15(4): 372-89$.
38. Watkins PB, Seeff LB. Drug-Induced Liver Injury: Summary of a Single Topic Clinical Research Conference. Hepatology. 2006;43(3):618-31.

39. Lucena MI, Andrade RJ, Kaplowitz N, García-Cortes M, Fernández MC, Romero-Gomez M, et al. Phenotypic characterization of idiosyncratic drug-induced liver injury: The influence of age and sex. Hepatology. 2009;49(6):2001-9.

40. Andrade, RJ, Lucena MI AA. HLA class II genotype influences the type of liver injury in drug-induced idiosyncratic liver disease. Hepatology. 2004;6(39):103-12.

41. Lammert C, Bjornsson E, Niklasson A, Chalasani N. Oral medications with significant hepatic metabolism at higher risk for hepatic adverse events. Hepatology. 2010;51(2):615-20.

42. García-Cortés M, Andrade RJ, Lucena MI, GonzálezGrande R, Camargo R, Fernández-Bonilla E, et al. Hepatotoxicidad secundaria a fármacos de uso común. Gastroenterol Hepatol. 2005;28(8):461-72.

43. Takikawa H. Assessment of 287 Japanese cases of drug induced liver injury by the diagnostic scale of the International Consensus Meeting. Hepatol Res. 2003;27(3):192-5.

44. Usui K, Oda Y, Kubota R, Negishi K, Uno K, Tsunematsu $\mathrm{S}$, et al. Clinical application of the leukocyte migration test and new diagnostic criteria for identifying causative agents in patients with drug-induced liver injury. Hepatogastroenterology. 2007;54(78):1752-7.

45. Food and Drug Administration F. Guidance for Industry Drug-Induced Liver Injury: Premarketing Clinical Evaluation. Center for Drug Evaluation and Research. 2009.

46. Lee WM. Drug-induced hepatotoxicity. N Engl J Med. 1995;333(17):1118-27.

47. Norris W, Paredes AH, Lewis JH. Drug-induced liver injury in 2007. Curr Opin Gastroenterol. 2008;24(3):289-97.

48. Bjornsson ES, Jonasson JG. Drug-induced cholestasis. Clin Liver Dis. 2013;17(2):191-209.

49. Suzuki A, Brunt EM, Kleiner DE, Miquel R, Smyrk TC, Andrade RJ, et al. The use of liver biopsy evaluation in discrimination of idiopathic autoinmune hepatitis vs. druginduced liver injury. Hepatology. 2011;54(3):931-939.

50. Degott D, Feldmann G, Larrey. Drug-induced prolonged cholestasis in adults: A histological semiquantitative study demonstrating progressive ductopenia. Hepatology. 1992;15(2): 244-51.

51. Daly P, Donaldson PT, Bhatnagar. HLA-B*5701 genotype is a major determinant of drug-induced liver injury due to flucloxacillin. Nat Genet. 2009;41(7):816-819.

52. O’Grady R, Alexander GJ, Hayllar KM, Williams R. Early indicators of prognosis in fulminant hepatic failure. Gastroenterol. 1989;97(2):439-495. 